\title{
Protective effect of fermented papaya preparation on stress-induced acute gastric mucosal lesion
}

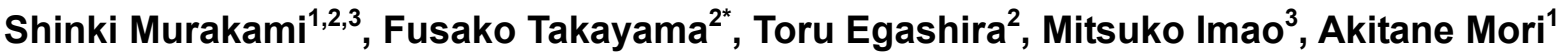 \\ ${ }^{1}$ Department of Brain Science, Graduate School of Medicine, Dentistry and Pharmaceutical Sciences, Okayama University, Okayama, \\ Japan \\ ${ }^{2}$ Department of Clinical Pharmaceutical Science, Graduate School of Medicine, Dentistry and Pharmaceutical Sciences, Okayama \\ University, Okayama, Japan; ${ }^{*}$ Corresponding Author: takayamf@cc.okayama-u.ac.jp \\ ${ }^{3}$ SAIDO Corporation, Fukuoka, Japan
}

Received 19 July 2012; revised 23 August 2012; accepted 3 September 2012

\begin{abstract}
Stress ulceration is single or multiple mucosal defects with/without bleeding from the gastric mucosa during the physiologic stress. Oxidative stress (OS) is a key pathogenic factor in psychogenic stress-induced acute gastric mucosal lesion (AGML). Fermented papaya preparation (FPP) is reported to have oxygen radical scavenging activity and is effective in OS-related diseases. Here, we investigated the protective effects and the mechanism of action of FPP on stressinduced AGML in rats, induced by water immersion restraint stress (WIRS). Exposure of rats to 6-hour WIRS resulted in the appearance of splinter hemorrhages and mucosal lesions in the stomach. WIRS induced significant increase in lipid peroxidation and decrease in superoxide dismutase-like activity in both the plasma and gastric mucosa. WIRS also significantly increased myeloperoxidase activity together with nuclear factor-kappaB (NF-kB) activation in gastric mucosa. FPP reduced all the above changes. The results suggest that oral administration of FPP provides protection against WIRS-induced AGML through its anti-oxidative and anti-inflammatory properties.
\end{abstract}

Keywords: Acute Gastric Mucosal Lesion; Fermented Papaya Preparation; Oxidative Stress; Inflammation

\section{INTRODUCTION}

Acute gastric mucosal lesion (AGML) is a common clinical problem. Although treatment for AGML is available, including $\mathrm{H}_{2}$ blockers, proton pump inhibitors, mucosal protectants, and decolonization of Helicobacter pylori, gastric mucosal lesion often relapse and the num- ber of patients afflicted with this entity has remained stable. Furthermore, the above treatments carry certain risks, such as side effect and resistant bacteria. Therefore, there is a need for more beneficial and preventive therapies for AGML.

Water immersion restraint stress (WIRS) is widely used as an experimental model of emotional stress-induced AGML based on its reproducibility and clinical relevance $[1,2]$. The underlying pathophysiology of WIRSinduced AGML is thought to be local tissue hypoxia and ischemia-reperfusion injury of the gastric mucosa associated with microvascular disturbances and infiltration of neutrophils, which induce subsequent inflammation by producing various proinflammatory mediators [2-4]. Under hypoxia and ischemia-reperfusion conditions, reactive oxygen species (ROS) are produced by the hypoxanthine-xanthine oxidase system and activated neutrophils in the gastric mucosa [4,5]. Accordingly, WIRS can induce an increase in lipid peroxidation and a decrease in reduced glutathione and superoxide dismutase contents in some organs, whereas treatment with reduced glutathione or superoxide dismutase and catalase can ameliorate WIRS-induced AGML [2,4-6]. Furthermore, under WIRS, the generated ROS may lead to inflammation and lesion formation in the gastric mucosa through the activation of nuclear factor-kappaB (NF-kB) [7]. Therefore, it is assumed that ROS may be generated during WIRS and could play an important role in the pathogenesis of stress-induced AGML.

Fermented papaya preparation (FPP) is a natural food prepared by fermentation of unripe papaya (Carica papaya Linn.) using several enzymes. Previous reports indicated that FPP has oxygen radical scavenging activity $[8,9]$ as well as potent and beneficial effects in oxidative stress (OS) related diseases, such as traumatic epilepsy [9, 10], Alzheimer's disease [11], and contact hypersensitivity [12]. To our knowledge, there is no information on the therapeutic effect of FPP in the diseases of the stomach. 
The aims of the present study were to determine the beneficial effects of orally administered FPP on WIRSinduced AGML, and to elucidate the possible mechanism of action.

\section{MATERIALS AND METHODS}

\subsection{Animals}

Male six-week-old Wistar rats weighting 160 - $170 \mathrm{~g}$ were purchased from Shimizu Experimental Animals (Shizuoka, Japan). The rats were housed in filter-protected cages set at $23^{\circ} \mathrm{C}$ and ambient light was controlled automatically to produce a light/dark $12 \mathrm{~h}$ cycle. The Animal Ethics Committee of Okayama University approved the study. All animal procedures described in this study were in strict accordance with the Guidelines for Animal Experiments at Okayama University Medical School.

\subsection{Experimental Design}

The animals were divided into various treatment groups. Rats of the Control group were provided with water alone $(n=4)$, whereas those of the WIRS group $(n=4)$ received glucose solution. FPP (SAIDO-PS501, SAIDO Co., Fukuoka, Japan) solution was administered orally at either low dose (100 mg/kg/day; group; $n=4)$ or high dose $(500 \mathrm{mg} / \mathrm{kg} / \mathrm{day} ; n=4)$ for 2 weeks before WIRS. Rats of WIRS + G500 mg/kg were received oral glucose solution $(500 \mathrm{mg} / \mathrm{kg} /$ day; $n=4)$ for 2 weeks before WIRS because FPP solution includes glucose. Cimetidine was administrated orally at $30 \mathrm{mg} / \mathrm{kg}$ (dissolved in carboxymetylcellulose sodium salt) at $30 \mathrm{~min}$ before WIRS (WIRS + cimetidine; $n=4$ ).

A gastric mucosal injury model induced by WIRS was prepared according to the method of Takagi and Okabe [1]. After fasting for $24 \mathrm{~h}$, rats were immobilized in a restraint cage and immersed in water up to the level of the xiphoid process in a temperature-controlled water $\left(23^{\circ} \mathrm{C}\right)$ for $6 \mathrm{~h}$. At the end of this period, they were immediately sacrificed under diethyl ether anesthesia; the stomach and blood were collected. The stomach was dissected along the greater curvature, and washed with ice-cold saline. The extent and areas of hemorrhagic erosion in the gastric body were examined and measured after imaging with digital camera. The percent area of the lesion, analyzed by Image $\mathrm{J}$ software, was designated as the Lesion index.

\subsection{Plasma and Gastric Mucosal Lipid Peroxidation Assay}

Diluted plasma and the supernatant collected from centrifugation of $30 \mathrm{mg} / \mathrm{ml}$ gastric mucosal homogenate in $120 \mathrm{mM} \mathrm{KCl}-30 \mathrm{mM}$ phosphate buffer ( $\mathrm{pH} 7.4)$, were mixed with Luminol $(130 \mu \mathrm{g} / \mathrm{ml})$ and analyzed in an Auto Lumat device (Tristar LB941, Berthold Technologies, Germany), at $37^{\circ} \mathrm{C}$ for $5 \mathrm{~min}$. Then, $0.33 \mathrm{mM}$ tertbutyl hydroperoxide $(t-\mathrm{BuOOH})$ was added, and the chemilumi-nescence (CL) intensity was detected for 120 min along by incubation at $37^{\circ} \mathrm{C}$. Data were expressed as mean \pm SEM of the cumulative CL intensity for 120 $\min$.

\subsection{Plasma and Gastric Mucosal Superoxide Dismutase-Like Activity}

Superoxide dismutase (SOD)-like activity was determined by electron paramagnetic resonance (ESR) spectroscopy. Superoxide anions were generated from hypoxanthine-xanthine oxidase system, and 5-(2,2dimethyl-1,3-propoxy cyclo-phosphoryl)-5-methyl-1-pyrroline $N$-oxide (CYPMPO; Radical Research, Tokyo) was used as spintrapping reagent. The gastric mucosa was homogenized in 9 volumes of ice-cold $50 \mathrm{mM}$ Tris- $\mathrm{HCl}$ buffer ( $\mathrm{pH} 7.5)$. The signal intensity was calculated by the ratio of the amplitude of the internal standard manganese signal and the CYPMPO-OOH spin adducts peak. The conditions for ESR spectrometric measurements were as follows: $331.5 \pm 10 \mathrm{mT}$ magnetic field; 8 $\mathrm{mW}$ power; receiver gain $5.0 \times 100$, modulation width 1.0 $\times 0.1 \mathrm{mT}$, time constant $0.1 \mathrm{sec}, \mathrm{A} / \mathrm{D}$ gain 3 , sweep time 4 min; room temperature.

\subsection{Myeloperoxidase Activity}

Myeloperoxidase (MPO) activity was used as an index of neutrophil infiltration in gastric mucosa. The gastric mucosa was homogenized in 9 volumes of ice-cold 0.3 $\mathrm{M}$ sucrose containing $0.22 \% \mathrm{CTAC} / 10 \mathrm{mM}$ citrate $(\mathrm{pH}$ $5.0)$ and sonicated. The homogenate was centrifuged for $20 \mathrm{~min}$ at $4^{\circ} \mathrm{C}$. The supernatant was reacted with substrate solution ( $3 \mathrm{mM}$ TMBZ, $120 \mu \mathrm{M}$ resorcinol, and 2.2 $\mathrm{mM} \mathrm{H}_{2} \mathrm{O}_{2}$ ) in shading and was mixed with $4 \mathrm{~N} \mathrm{H}_{2} \mathrm{SO}_{4}$, then, absorbance was read at $450 \mathrm{~nm}$.

\subsection{Western Blot Analysis}

Gastric mucosal NF- $\mathrm{BB}$ expression was analyzed by Western blot method. The gastric mucosal nuclear was solubilized with sodium dodecyl sulfate (SDS) in $0.5 \mathrm{M}$ Tris- $\mathrm{HCl}(\mathrm{pH}$ 6.8) 10\% glycerol, 5\% 2-mercaptoethanol and $0.05 \%$ bromophenol blue, and then degenerated by incubation at $100^{\circ} \mathrm{C}$ for $5 \mathrm{~min}$. Samples were subjected to SDS-10\% polyacrylamide gel electrophoresis. After electrophoresis, proteins were transferred onto polyvinylidene fluoride (PVDF) membrane. Then, the non-specific binding proteins were blocked using 5\% nonfat milk dissolved in TBS-T (Tween20 in Tris-buffered saline, $\mathrm{pH}$ 7.4) for 1 hour and was the membrane was incubated with the primary antibody (mouse anti-human/ 
rat NF- $\kappa$ B monoclonal antibody) for 1 hour, and subsequently incubated with the secondary anti-mouse $\operatorname{IgG}$ antibody (1:5000; Santa Cruz Biotechnology, Santa Cruz, CA) for 1 hour. Histone was used to ensure equal protein loading. Protein bands were visualized using enhanced Chemiluminescence Luminol Reagent (Santa Cruz Biotechnology).

\subsection{Statistical Analysis}

Values were expressed as mean \pm standard error of the mean (SEM) of 4 - 6 rats. Differences among groups were examined by one-way analysis of variance (ANOVA) with Tukey test. A $P$ value less than 0.05 was considered statistically significant.

\section{RESULTS}

\subsection{Effects of FPP on Stress-Induced Gastric Mucosal Lesion}

The effects of FPP, glucose and cimetidine on WIRSinduced gastric mucosal lesion are shown in Figure 1 and Table 1 Marked splinter bleeding and mucosal lesions were found in the glandular part of the stomach in WIRS rats. Administration of FPP $100 \mathrm{mg} / \mathrm{kg} /$ day and glucose $500 \mathrm{mg} / \mathrm{kg} /$ day did not produce any significant protection, whereas administration of FPP $500 \mathrm{mg} / \mathrm{kg} /$ day and cimetidine $30 \mathrm{mg} / \mathrm{kg}$ resulted in a significant improvement of bleeding and mucosal damage.

Scheme: experimental design
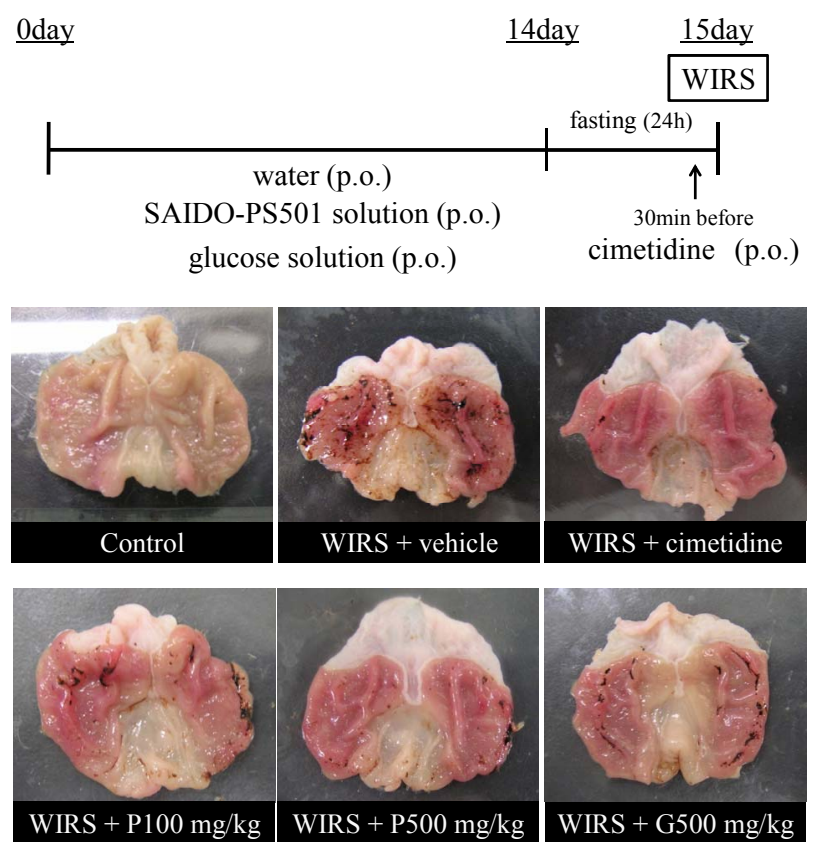

Figure 1. Effects of FPP, glucose and cimetidine on WIRSinduced lesions in the gastric mucosa (macroscopic view).
Table 1. Effects of FPP, glucose and cimetidine on WIRS-induced lesions in the gastric mucosa. Lesion Index was calculated as lesion area per whole area $\times 100(\%)$.

\begin{tabular}{cc}
\hline Lesion index $(\%$ : lesion area/whole area $\times 100)$ \\
\hline Control & $0.00 \pm 0.00$ \\
WIRS + vehicle & $3.62 \pm 0.39^{* *}$ \\
WIRS + cimetidine & $0.95 \pm 0.23^{\#}$ \\
WIRS + P $100 \mathrm{mg} / \mathrm{kg}$ & $2.26 \pm 0.44$ \\
WIRS + P $500 \mathrm{mg} / \mathrm{kg}$ & $1.06 \pm 0.39^{\#}$ \\
WIRS + G $500 \mathrm{mg} / \mathrm{kg}$ & $3.53 \pm 0.71$ \\
\hline
\end{tabular}

\subsection{Effects of FPP on Plasma and Gastric Mucosal Lipid Peroxidation}

WIRS was associated with significant increases in plasma and gastric mucosal lipid peroxidation compared with the control rats $(P<0.01$, Figures 2(a) and (b)). Treatment with FPP $500 \mathrm{mg} / \mathrm{kg}$ reduced both the plasma $(P<0.05$, Figure 2(a)) and gastric mucosal $(P<0.05$, Figure 2(b)) lipid peroxidation compared with WIRS rats.

\subsection{Effects of FPP on Plasma and Gastric Mucosal SOD-Like Activity}

WIRS significantly reduced the plasma level of SODlike activity compared with the control $(P<0.05$, Figure 3(a)). Administration of FPP $500 \mathrm{mg} / \mathrm{kg}$ reversed plasma SOD-like activity $(P<0.05$, vs WIRS). WIRS also decreased the gastric mucosal SOD-like activity $(P<0.05$, Figure 3(b)), but FPP administration had no significant effect on the activity $(P>0.05)$.

\subsection{Effects of FPP on MPO Activity}

MPO activity represented an index of tissue neutrophil infiltration. WIRS significantly increased gastric mucosal MPO activity relative to the control $(P<0.05$, Figure 4), and such increase was significantly reduced by FPP $500 \mathrm{mg} / \mathrm{kg}(P<0.05)$.

\subsection{Effects of FPP on NF-KB Activation}

Within 6 hours, WIRS significantly increased the nuclear translocation of NF- $\kappa \mathrm{B}$ in the gastric mucosa $(P<$ 0.01 , Figure 5). Administration of FPP at $500 \mathrm{mg} / \mathrm{kg}$

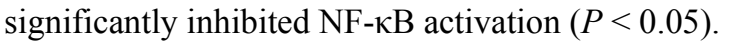

\section{DISCUSSION}

The presents study demonstrated that oral administration of FPP attenuated the development of gastric mucosal lesion in rats subjected to WIRS. Although the pathogenesis of stress-induced acute gastric mucosal lesion 
AGML is not fully understood, evidence suggests the involvement of OS in this process. WIRS is widely used as an experimental model of emotional stress-induced AGML based on its reproducibility and clinical relevance $[1,2]$. Using this model, it has been reported that

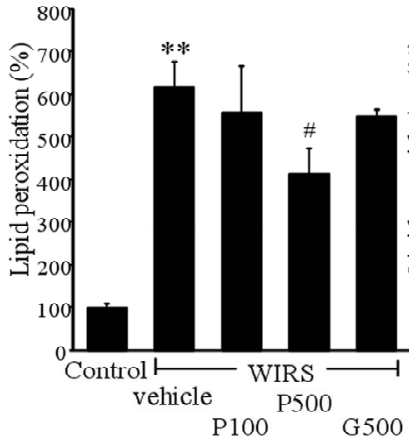

(a)

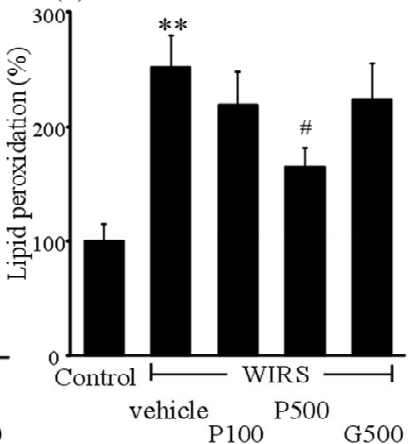

(b)
Figure 2. Effects of FPP on plasma lipid peroxidation (a) and gastric mucosal lipid peroxidation (b). Data are mean \pm SEM of $4-6$ rats. ${ }^{* *} P<0.01$ vs. Control; ${ }^{\#} P<0.05$ vs. WIRS + vehicle (ANOVA with Tukey-test).

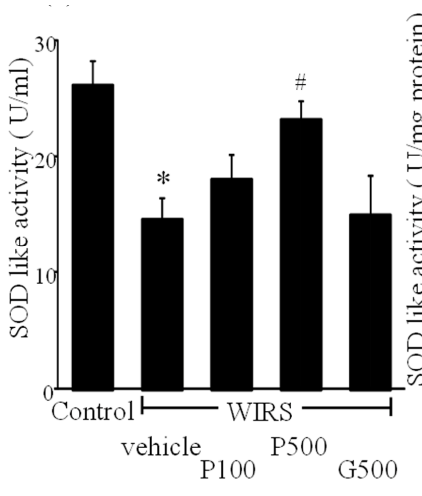

(a)

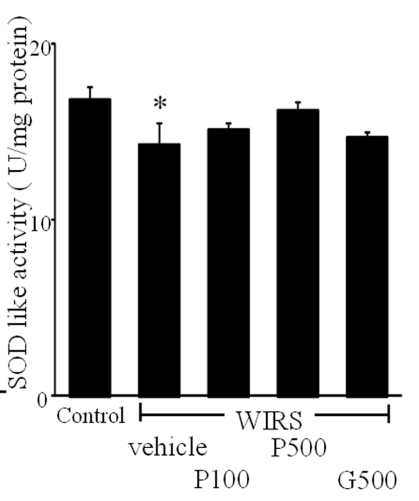

(b)
Figure 3. Effects of FPP on plasma SOD-like activity (a) and gastric mucosal SOD-like activity (b). Data are mean \pm SEM of 4 - 6 rats. ${ }^{*} P<0.05$ vs. Control; ${ }^{\#} P<0.05$ vs. WIRS + vehicle (ANOVA with Tukey-test)

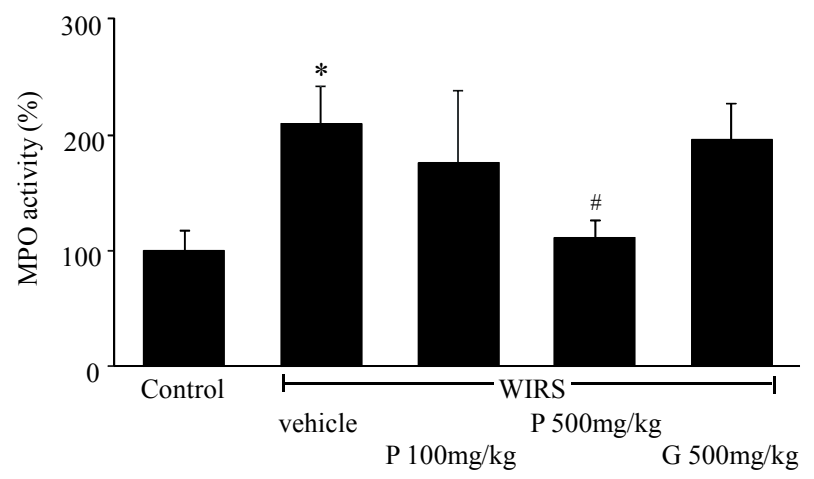

Figure 4. Effects of FPP on gastric mucosal MPO activity. Data are mean \pm SEM of $4-6$ rats. ${ }^{*} P<0.05$ vs. Control; ${ }^{\#} P<0.05$ vs. WIRS + vehicle (ANOVA with Tukey-test).

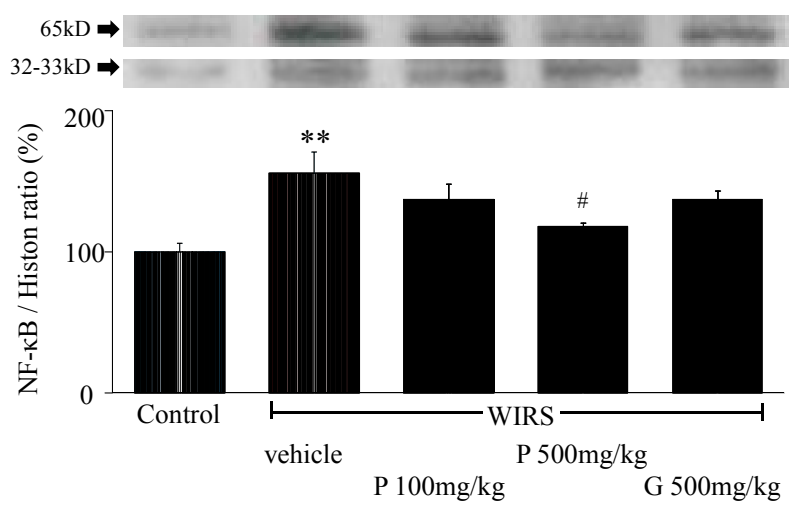

Figure 5. Effects of FPP on nuclear translocation of NF- $\kappa \mathrm{B}$ in gastric mucosa. Data are mean \pm SEM of 4 rats. ${ }^{* *} P<0.01$ vs. Control; ${ }^{\#} P<0.05$ vs. WIRS + vehicle (ANOVA with Tukeytest).

OS increases ROS, free radicals and lipid peroxides and/or decreases antioxidant components, and thus plays an important role in the pathogenesis of AGML [4-6]. The mechanism is assumed to relate to ischemia or hypoxemia, which can induce increased generation of free radicals through the xanthine-xanthine oxidase system and infiltrated neutrophils in the gastric mucosa. Xanthine oxidase produces superoxide anions and $\mathrm{H}_{2} \mathrm{O}_{2}$ by catalyzing the oxidation of hypoxanthine or xanthine using $\mathrm{O}_{2}$ as the electron acceptor. High levels of xanthine oxidase activity and high serum uric acid (xanthine oxidase-derived metabolite) concentrations have been found in rats exposed by WIRS [4,5]. In the present study, we showed that 6 hour of WIRS provoked an increase in lipid peroxidation and a decrease in SOD-like activity in both serum and gastric mucosa. Moreover, administration of FPP ameliorated stress-induced gastric lesion formation, accompanied by a decrease in lipid peroxidetion and an increase in SOD-like activity. Generally, antioxidants are known to prevent the progression of gastric mucosal injury [5,6,13-16]. On the other hand, FPP is known as a radical scavenger against superoxide anions and hydroxyl radicals in vitro [8,9]. Thus, oral administration of FPP could prevent WIRS-induced AGML by suppressing OS.

Inflammatory response is also known to be an important pathway in the development of AGML. NADPH oxidase is present in the membranes of neutrophils, which seems to be the major source of superoxide radical anion. Neutrophils could play a crucial role in WIRSinduced gastric mucosal injury, including generation of oxygen free radicals. We measured MPO activity in gastric mucosa as an index of neutrophil infiltration into the gastric mucosa. In the present study, MPO activity was significantly increased in WIRS rats, and administration of FPP reduced such activity. Thus, one can conclude 
that FPP can reduce neutrophils accumulation and subsequent ROS production in the gastric mucosa, and thus it can suppress gastric mucosal injury.

$\mathrm{NF}-\kappa \mathrm{B}$ is a transcription factor known to induce various genes involved in physiological processes as well as those related to the response to injury and infection [17]. Activation of $N F-\kappa B$ in the cytoplasm followed by its translocation to the nucleus is controlled by targeted phosphorylation and subsequent degradation of I-kappa B

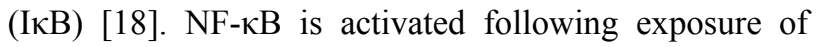
cells to lipopolysaccharides, inflammatory cytokines (e.g., TNF and IL-1), viral infection and OS. NF- $\kappa$ B can induce the transcription of proinflammatory cytokines, chemokines, adhesion molecules, matrix metalloproteinase, Cox-2, and inducible nitric oxide synthase. Experimental evidence indicates that free radical scavengers inhibit WIRS-induced gastric mucosal injury through the suppression of NF- $\kappa B$ activation [7]. Hence, OS is thought to activate NF- $\kappa \mathrm{B}$ through phosphorylation and degradation of I $\mathrm{BSs}$ in the cytoplasm of gastric mucosal cells in rats exposed to WIRS. In support of this notion, the present study showed that WIRS induced a significant increase in NF- $\kappa \mathrm{B}$ nuclear translocation in the gastric mucosa, and that FPP administration prevented these changes.

In conclusion, the present study demonstrated the beneficial effects of FPP against WIRS-induced AGML, and that these effects probably involve the anti-oxidative and anti-inflammatory properties of FPP.

\section{REFERENCES}

[1] Takagi, K. and Okabe, S. (1968) The effects of drugs on the production and recovery processes of the stress ulcer. The Japanese Journal of Pharmacology, 18, 9-18. doi:10.1254/jijp. 18.9

[2] Kwiecień, S., Brzozowski, T. and Konturek, S.J. (2002) Effects of reactive oxygen species action on gastric mucosa in various models of mucosal injury. Journal of Pysiology and Pharmacology, 52, 39-50.

[3] Hamaguchi, M., Watanabe, T., Higuchi, K., Tominaga, K. Fujiwara, Y. and Arakawa, T. (2001) Mechanisms and roles of neutrophil infiltration in stress-induced gastric injury in rats. Digestive Diseases and Science, 46, 2708-2715. doi:10.1023/A:1012779530004

[4] Nishida, K., Ohta, Y., Kobayashi, T. and Ishiguro, I. (1997) Involvement of the xanthine-xanthine oxidase system and neutrophils in the development of acute gastric mucosal lesions in rats with water immersion restraint stress. $\mathrm{Di}$ gestion, 58, 340-351.

[5] Ohta, Y. and Nishida, K. (2003) Protective effect of coadministered superoxide dismutase and catalase against stressinduced gastric mucosal lesions. Clinical and Experimental Pharmacology and Physiology, 30, 545-550. doi:10.1046/j.1440-1681.2003.03871.x

[6] Hirota, M., Inoue, M., Ando, Y., Hirayama, K., Morino, Y.,
Sakamoto, K., Mori, K. and Akagi, M. (1989) Inhibition of stress-induced gastric injury in the rat by glutathione. Gastroenterology, 97, 853-859.

[7] Jia, Y.T., Ma, B., Wei, W., Xu, Y., Wang, Y., Tang, H.T. and Xia, Z.F. (2007) Sustained activation of nuclear factor$\kappa \mathrm{B}$ by reactive oxygen species is involved in the pathogenesis of stress-induced gastric damage in rats. Critical Care Medicine, 35, 1582-1591. doi:10.1097/01.CCM.0000266824.82280.17

[8] Noda, Y., Murakami, S., Mankura, M. and Mori, A. (2008) Inhibitory effect of fermented papaya preparation on hydroxyl radical generation from methylguanidine. Journal of Clinical Biochemistry and Nutrition, 43, 185-190. doi:10.3164/jcbn.2008062

[9] Imao, K., Wang, H., Komatsu, M. and Hiramatsu, M. (1998) Free radical scavenging activity of fermented papaya preparation and its effect on lipid peroxide level and superoxide dismutase activity in iron-induced epileptic foci of rats. Biochemistry and Molecular Biology International, 45, 11-23.

[10] Imao, K., Komatsu, M., Wang, H. and Hiramatsu, M. (1999) Inhibitory effect of fermented papaya preparation on oxidative DNA damage and tissue injury in the brain formed during iron-induced epileptogenesis in rats. Journal of Brain Science, 25, 71-77.

[11] Zhang, J., Mori, A., Chen, Q. and Zhao, B.L. (2006) Fermented papaya preparation attenuates $\beta$-amyloid precursor protein: $\beta$-amyloid-mediated copper neurotoxicity in $\beta$-amyloid precursor protein and in $\beta$-amyloid precursor Swedish mutation overexpressing SH-SY5Y cells. Neuroscience, 143, 63-72.

doi:10.1016/j.neuroscience.2006.07.023

[12] Hiramoto, K., Imao, M., Sato, E., Inoue, M. and Mori, A. (2008) Effect of fermented papaya preparation on dermal and intestinal mucosal immunity and allergic inflammations. Journal of the Science of Food and Agriculture, $\mathbf{8}$, 1151-1157. doi:10.1002/jsfa.3190

[13] Ohta,Y., Imai, Y., Kaida, S., Kamiya, Y., Kawanishi, M. and Hirata, I. (2010) Vitamin E protects against stressinduced gastric mucosal lesions in rats more effectively than vitamin C. Biofactors, 36, 60-69.

[14] Abdallah, D.M., El-Abhar, H.S. and Abdel-Aziz, D.H. (2009) TEMPOL, a membrane-permeable radical scavenger, attenuates gastric mucosal damage induced by ischemia/ reperfusion: A key role for superoxide anion. European Journal of Pharmacology, 603, 93-97. doi:10.1016/j.ejphar.2008.11.057

[15] Ohta, Y., Kobayashi, T., Nishida, K., Nagata, M. and Ishiguro, I. (1999) Therapeutic effect of Oren-gedoku-to extract on stress-induced acute gastric mucosal lesions in rats. Phytotherapy Research, 13, 588-592.

doi:10.1002/(SICI)1099-1573(199911)13:7<588::AID-P TR505>3.0.CO;2-P

[16] Iwasaki, Y., Matsui, T. and Arakawa, Y. (2004) The protective and hormonal effects of proanthocyanidin against gastric mucosal injury in wistar rats. Journal of Gastroenterology, 39, 831-837. doi:10.1007/s00535-004-1399-5

[17] Bowie, A, O’Neill L.A. (2000) Oxidative stress and nuclear factor- $\kappa \mathrm{B}$ activation: A reassessment of the evidence in 
the light of recent discoveries. Biochemical Pharmacology, 59, 13-23. doi:10.1016/S0006-2952(99)00296-8

[18] Baldwin, A.S. Jr. (1996) The NF-kappa B and I kappa B pro- teins: New discoveries and insights. Annual Reviews Immunology, 14, 649-683. 